\title{
Equilibriums in Logistic Chain with Various Optimization Criteria of Participants
}

\author{
Valery Heyets \\ Institute for Economics and \\ Forecasting \\ Kyiv, Ukraine \\ heyets@nas.gov.ua \\ http://orcid.org/0000-0002-2895-6114
}

\author{
Mykhailo Zvieriakov \\ Odesa National Economic University \\ Odesa, Ukraine \\ mail@oneu.edu.ua \\ http://orcid.org/0000-0003-0039-5068
}

\author{
Anatoliy Kholodenko \\ Deparment of Business and Tourism \\ Odesa National Maritime University \\ Odesa, Ukraine \\ anathol2035@gmail.com \\ https://orcid.org/0000-0001-7626-5820
}

\begin{abstract}
The logistic chain of production and delivery of products on the market, including manufacturing and transport enterprises, is considered. Equilibrium states in this logistic chain for various combinations of participant's optimization criteria, such as profit, gross income, cargo turnover, profitability, profit intensity, are analytically found and compared. The asymptotic behavior of equilibrium transport tariffs in this system is investigated and presented.
\end{abstract}

Keywords - logistic chain, equilibrium states, optimization criteria, transport tariffs, asymptotic behavior.

\section{INTRODUCTION}

The researches of logistics chains that are linking producers, carriers and consumers of products, forecasting of their expected behavior are becoming of particularly great value at the present stage of market transformation and the development of transport corridors.

However, in numerous literature on logistics, which has been extensively published in recent years [1, 2], in particular the integrated supply chains [3-8], relevant issues are considered primarily on the descriptive level, the best case scenario with the simplest calculation formulas. From our point of view, the study of logistics systems with the use and development of relevant results in microeconomics [912] and the theory of organization of industrial markets [1316] deserves serious attention.

In addition, forecasting of the logistic chains' behavior by finding their equilibrium states deviating from which is not beneficial to any of the participants is of considerable interest. This approach to forecasting based on determining and modeling of meaningful relationships between enterprises in all their diversity and depth, rather than the formal processing of time series statistics, is considered as more appropriate and promising. It should be noted that the participants in the logistics chains can have both traditional equal criteria for maximizing own profit and to pursue different objectives such as maximizing income, goods turnover, profitability, intensity of profit, etc.

Therefore, the purpose of this article is to establish and compare equilibriums in the logistics chain for different combinations of criteria for participants' optimality, because the chain's staying exactly in one of the following equilibrium conditions can be forecasted.

Let the logistic chain (Fig. 1) includes the consumer market $\mathbf{M}$ where price is formed as $P=b-k Q$, a producer $\mathbf{P}$ with the unit cost $\mathcal{V}$ and a transport company $\mathbf{T}$ with the cost for transportation of each unit of production $Z$, where $\boldsymbol{b}>\mathbf{O}$ - is the maximum possible price of products in the market; $\boldsymbol{k}>\mathbf{O}$ is the indicator of demand's elasticity (price reduction with the increase in output) on the consumer market; $Q \geq 0$ - volume of the production and transportation of products to the market.

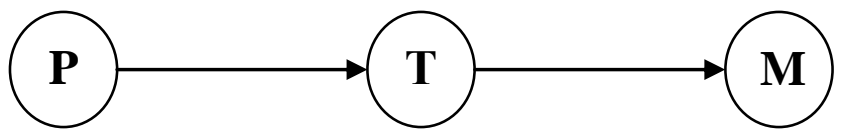

Fig. 1. Fig. 1. Investigated logistic chain

\section{COMBINATION OF CRITERIA PROFIT - PROFIT}

Let us suppose first that each of the participants (1producer, 2-carrier) maximizes their profits:

$$
\begin{aligned}
& F_{1}=P Q-v Q-p Q=((b-k Q)-v-p) Q \underset{Q}{\longrightarrow} \max \\
& F_{2}=p Q-z Q=(p-z) Q \underset{p}{\longrightarrow} \max ,
\end{aligned}
$$

where $p$ is the tariff for production unit transportation.

Let us equate a derivative from the function of producer's profit with a zero

$$
\frac{d F_{1}}{d Q}=b-2 k Q-v-p=0
$$

It follows that the function of producer's optimal reaction on the present transport tariff has the next form:

$$
Q=\frac{b-v-p}{2 k}
$$

Let us put this expression in (2):

$$
F_{2}=(p-z)\left(\frac{b-v-p}{2 k}\right) \underset{p}{\longrightarrow} \max
$$

hence

$$
\frac{d F_{2}}{d p}=\frac{b-v-p}{2 k}+(p-z) \frac{-1}{2 k}=\frac{b-v+z-2 p}{2 k}=0
$$

equilibrium transport tariff

$$
p^{F F}=\frac{b-v+z}{2}=z+\frac{b-v-z}{2}=z+\frac{A}{2}
$$


Here the top index $F F$ means the balance $F \boldsymbol{F}$, when each of the participants of the production and transport chain maximizes its own profit; $\boldsymbol{A}=\boldsymbol{b}-\boldsymbol{v}-\boldsymbol{z}>\mathrm{O}$ is the economic potential of the production and transport chain (if it is a negative, then producing of products and their delivery to the market makes no sense). Equilibrium transport tariff consists of transportation costs and half of economic potential of the chain.

Substituting (5) in (3), we obtain the equilibrium amount of products

$$
Q^{F F}=\frac{b-v-z}{4 k}=\frac{A}{4 k} .
$$

Equilibrium market price

$$
P^{F F}=\frac{3 b+v+z}{4}=b-\frac{A}{4},
$$

equilibrium participant's profits

$$
\begin{aligned}
& F_{1}^{F F}=\frac{(b-v-z)^{2}}{16 k}=\frac{A^{2}}{16 k}, \\
& F_{2}^{F F}=\frac{(b-v-z)^{2}}{8 k}=\frac{A^{2}}{8 k},
\end{aligned}
$$

total equilibrium profit

$$
F^{F F}=F_{1}^{F F}+F_{2}^{F F}=\frac{3 A^{2}}{16 k} .
$$

\section{COMBINATION OF CRITERIA PROFIT - INCOME}

If now the transport enterprise maximizes gross income instead of profit, then its criterion (2) converts into

$$
D_{2}=p Q \longrightarrow \text { max . }
$$

Substituting here the producer's reaction function (3), which as before maximizes profits, we obtain

$$
D_{2}=p \frac{b-v-p}{2 k} \underset{p}{\longrightarrow} \max
$$

hence

$$
\frac{d D_{2}}{d p}=\frac{b-v-p}{2 k}+p \frac{-1}{2 k}=\frac{b-v-2 p}{2 k}=0,
$$

the equilibrium transport tariff

$$
p^{F D}=\frac{b-v}{2}=z+\frac{b-v-2 z}{2}=\frac{z}{2}+\frac{A}{2} .
$$

It is easy to see that $p^{F F}-p^{F D}=\frac{z}{2}>0$. This means that if a transport enterprise will maximize gross profit instead of profit, its equilibrium tariff will drop to half of the transportation cost.

Herewith the equilibrium amount of products

$$
Q^{F D}=\frac{b-v}{4 k}=\frac{A}{4 k}+\frac{z}{4 k}
$$

increases by $\frac{z}{4 k}$ in comparison with $Q^{F F}$, equilibrium market price $\quad P^{F D}=\frac{3 b+v}{4}=b-\frac{A}{4}-\frac{z}{4}$ reduces by $\frac{z}{4}$ (a quarter of transportation cost) in comparison with the $P^{F F}$. Equilibrium profits of participants $\quad F_{1}^{F D}=\frac{(b-v)^{2}}{16 k}>F_{1}^{F F}$ $F_{2}^{F D}=\frac{(b-v-2 z)(b-v)}{8 k}<F_{2}^{F F}$, total equilibrium profit of production and transport chain participants

$$
F^{F D}=F_{1}^{F D}+F_{2}^{F D}=\frac{3(b-v)^{2}-4 z(b-v)}{16 k} .
$$

It is easy to see that

$$
\begin{gathered}
F_{2}^{F D}>0 \text { if } z<\frac{b-v}{2}, \\
F^{F D}>F^{F F} \text { when } z<\frac{2(b-v)}{3}, \\
F^{F D}<F^{F F} \text { if } z>\frac{2(b-v)}{3},
\end{gathered}
$$

that is, when the transportation costs low enough (in any case, such, that provides to the transport enterprise positive profits even when it maximizes not profit, but revenue) total profit of participants increases from such a transport enterprise's criterion of optimality change.

\section{COMBINATION OF CRITERIA PROFIT - CARGO TURNOVER}

If we assume that a transport enterprise maximizes neither profit nor gross income, but an amount of transported products (cargo turnover), which means that the criterion

$$
Q=\frac{b-v-p}{2 k} \underset{p}{\longrightarrow} \max ,
$$

hence $p^{F Q}=0$, the equilibrium volume of production $Q^{F Q}=\frac{b-v}{2 k}=\frac{A}{2 k}+\frac{z}{2 k} \quad$ increases twofold even compared with $Q^{F D}$, the equilibrium producer's profit $F_{1}^{F Q}=\frac{(b-v)^{2}}{4 k}$, losses of transport enterprise $F_{2}^{F Q}=-z \frac{b-v}{2 k}$, the total profit of participants $F^{F Q}=F_{1}^{F Q}+F_{2}^{F Q}=\frac{4(b-v)^{2}-8 z(b-v)}{16 k}$. It is easy to see that $F^{F Q}>F^{F D}>F^{F F}$ in 
case of sufficiently low transportation cost $z<\frac{b-v}{4}$, when $z>\frac{b-v}{4}$ will take place $F^{F Q}<F^{F D}$.

We will note that even larger maximum total profit of the participants will be achieved with their integration (set by index $i$ ), when the transport tariff will be not zero, and the cost of transportation: when $p=z$ get $Q^{i}=\frac{A}{2 k}$,

$$
\begin{gathered}
P^{i}=b-\frac{A}{2}, F^{i}=\frac{A^{2}}{4 k}, \\
F^{i}-F^{F Q}=\frac{z^{2}}{4 k}>0 .
\end{gathered}
$$

\section{COMBINATION OF CRITERIA PROFIT - PROFITABILITY}

If a transport company maximizes its own profitability, then its fixed costs $C$ should be considered too. Then function of profitability $R_{2}=\frac{F_{2}}{Z_{2}}=\frac{(p-z) Q}{z Q+c}$, substituting for it function of the reaction of the producer (3), we obtain

$$
R_{2}=\frac{(p-z) \frac{b-v-p}{2 k}}{z \frac{b-v-p}{2 k}+c} \underset{p}{\longrightarrow} \max
$$

After equating to zero derivative $\frac{d R_{2}}{d p}$ and solving the appropriate equation, we find the equilibrium transport tariff $p^{F R}=b-v+\frac{2 k c-\sqrt{4 k^{2} c^{2}+2 z k c(b-v-z)}}{z}$ It is interesting that $\frac{d p^{F R}}{d c}<0$, that is with transport enterprise's fixed costs increasing its optimal by a criterion of profitability tariff falls (the conclusion at first glance paradoxical but, in fact, for large fixed costs enterprise even when the criterion is profitability has to think not about the norm, but about the mass of profit to cover these fixed costs), and slower, because $\frac{d^{2} p^{F R}}{d c^{2}}>0$, that is the function $p^{F R}(c)$ convex downwards.

Since $\quad \lim _{c \rightarrow \infty} p^{F R}=\frac{b-v+z}{2}=p^{F F}$ optimal by a criterion of profitability transport tariff falls not to zero, but asymptotically approaches optimal by a criterion of maximum profit of the transport company tariff.
It follows that if a transport company maximizes profitability of transportation, equilibrium transport tariff will increase in comparison with the basic equilibrium $\boldsymbol{F F}$, accordingly transport volumes and total profits of the participants will reduce.

\section{COMBINATION OF CRITERIA PROFIT - PROFIT INTENSITY}

Finally, let us consider the current case when a transport enterprise, combining a financial factor with a time factor, maximizes the intensity of profit as the ratio of its profit not to cost (as in just considered criterion of profitability) but the length of time used for receiving this profit [17]. We shall enter here value $a$ as fixed time expenditures on cargo transportation (clearance), that do not depend on its volume $Q$, and capacity of transport enterprise $\boldsymbol{M}$ as the amount of cargo carried per unit of time.

Then the intensity of transport enterprise's profit $I_{2}=\frac{F_{2}}{T}=\frac{(p-z) Q}{a+\frac{Q}{v}}$, substituting for it function of the producer's reaction on the transport tariff and turning it, we obtain

$$
\begin{aligned}
& I_{2}=M \frac{(p-z) \frac{b-v-p}{2 k}}{a M+\frac{b-v-p}{2 k}} \underset{p}{\longrightarrow} \max \\
& \text { Equating to zero derivative } \frac{d I_{2}}{d p} \text { and solving the }
\end{aligned}
$$
appropriate equation, we find the equilibrium transport tariff $p^{F I}=b-v+2 a M k-\sqrt{4 a^{2} M^{2} k^{2}+2 a M k(b-v-z)}$.

Similar to the previous analysis by the criterion of profitability, $\frac{d p^{F I}}{d a}<0$, that is with transport enterprise's constant time expenditures increasing its optimum by a criterion of profit's intensity tariff falls, and slower, because $\frac{d^{2} p^{F I}}{d a^{2}}>0$, that is the function $p^{F I}(a)$ convex downwards.

Since $\quad \lim _{a \rightarrow \infty} p^{F I}=\frac{b-v+z}{2}=p^{F F}$, optimal by criterion of profit's intensity transport tariff falls not to zero, but asymptotically approaches optimal by a criterion of maximum profit of the transport company's tariff.

Thus, if a transport company (seaport) instead of profit begins to maximize the intensity of the profits, its tariff increases, the volume of traffic or transshipment (batch of 
products) reduces, profit from each batch of products decreases as the intensity of the profit (per unit of time) increases.

\section{COMBINATION OF CRITERIA PROFIT INTENSITY - PROFIT}

Let us now explore a situation when a producer maximizes not the profit but the intensity of the profit which is the profit per unit of time:

$$
I_{1}=\frac{F_{1}}{t}=\frac{((b-k Q)-p-v) \cdot Q}{a+\frac{Q}{M}} \rightarrow \max _{Q}
$$

where $t$ is the total time of transport and sales of products batch;

$a$ - conditionally constant time for transportation and sales that do not depend on the amount of a product batch $Q$;

$M$ - capacity (volume of products' transportation per unit of time).

Let us equate to zero the first derivative of the profit's intensity function:

$$
\frac{d I_{1}}{d Q}=M \frac{(b-2 k Q-p-v) \cdot(a M+Q)-((b-k Q)-p-v) \cdot Q}{(a M+Q)^{2}}=0,
$$

from here we get a square equation concerning $Q$ :

$$
k Q^{2}+2 a k M Q-a M(b-p-v)=0,
$$

which solution gives us the function of producer's optimal reaction to the current transport tariff $p$

$Q^{I F}=\sqrt{a^{2} M^{2}+\frac{a M(b-p-v)}{k}}-a M=a M\left(\sqrt{1+\frac{b-p-v}{a k M}}-1\right)$

Let us explore a behavior of this function with arguments $\boldsymbol{a}$ and $\boldsymbol{M}$, determining the time of transportation (a factor which is the object of study in this article), with fixed values of the rest of arguments. We can show that $\frac{\partial Q^{I F}}{\partial a}>0$ and $\frac{\partial Q^{I F}}{\partial M}>0$, that is, the more conditionally constant transportation and sale time of products batch and the greater transport capacity, the greater should be the volume of this product batch (which is consistent with the economic sense - if the organization of transportation is long compared with the transportation itself, then you should carry large batches)

\section{Asymptotically}

$\lim _{a \rightarrow \infty} Q^{I F}=\lim _{M \rightarrow \infty} Q^{I F}=\frac{b-p-v}{2 k}$, that is when the infinite time of organizing of products transportation the function of the producer's reaction by intensity of profits criterion (12) no longer depends neither on $\boldsymbol{a}$, nor on $\boldsymbol{M}$, and will match with the function of the producer's reaction by profit criterion. Determined principle view of the dependence $Q^{I F}(a)$ is shown in Fig.2.

It is easy to show that the dependence of the production volume $Q^{I F}$ on the transport capacity $M$ will also have the same principle view - the optimal volume of products will increase slower with the increasing of its transportation capabilities per unit of time.

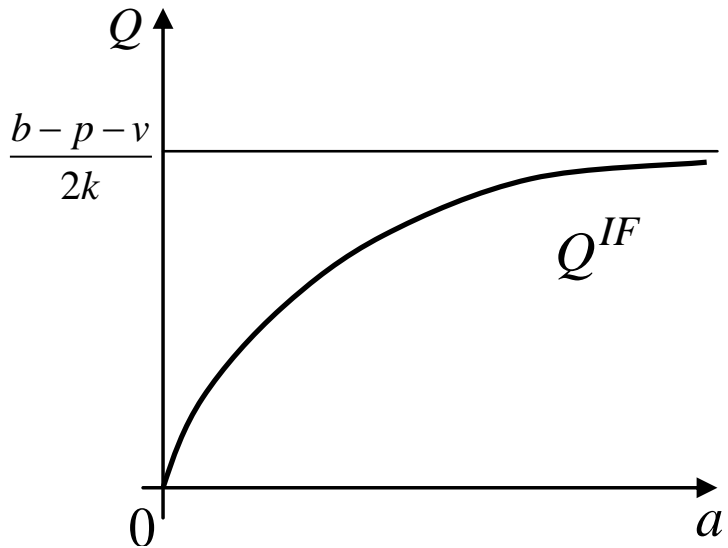

Fig. 2. Principal view of the production volume's $Q^{I F}$ dependence on conditionally constant time for transportation and sale $a$

Substituting production volume $Q^{I F}$ (12) to the function of the manufacturer's profit (11), we obtain

$F_{1}^{I F}=(b-p-v) \cdot\left(\sqrt{a^{2} M^{2}+\frac{a M(b-p-v)}{k}}-a M\right)-$

$-k\left(2 a^{2} M^{2}+\frac{a M(b-p-v)}{k}-2 a M \sqrt{a^{2} M^{2}+\frac{a M(b-p-v)}{k}}\right)$.

In this case

$\lim _{a \rightarrow \infty} F_{1}^{I F}=\lim _{M \rightarrow \infty} F_{1}^{I F}=\frac{(b-p-v)^{2}}{4 k}$, that matches the producer's profit in equilibrium $F \boldsymbol{F}$.

Therefore, dependences of the producer's profit $F_{1}^{I F}(a)$ and $F_{1}^{I F}(M)$ on parameters that determine the time of transportation have the same principal view as in Fig.1.

The influence of the same parameters $\boldsymbol{A}$ and $\boldsymbol{M}$ on transportation time

$$
t^{I F}=a+\frac{Q^{I F}}{M}=\sqrt{a^{2}+\frac{a(b-p-v)}{k M}}
$$

is different. 
Denoting $c=\frac{b-p-v}{k M}>0$, we obtain

$$
\begin{gathered}
t^{I F}=\sqrt{a^{2}+a c}, \\
\frac{\partial t^{I F}}{\partial a}=\frac{2 a+c}{2 \sqrt{a^{2}+a c}}>0, \\
\frac{\partial^{2} t^{I F}}{\partial a^{2}}=\frac{-c^{2}}{4 \sqrt{\left(a^{2}+a c\right)^{3}}}<0,
\end{gathered}
$$

that is the function $t^{I F}(a)$ with a fixed $M$ rising and convex upwards.

Inclined asymptote of the function $t^{I F}(a)$ has form $t=c_{1} a+c_{2}$, where

$$
\begin{gathered}
c_{1}=\lim _{a \rightarrow \infty} \frac{t^{I F}}{a}=\lim _{a \rightarrow \infty} \frac{\sqrt{a^{2}+a c}}{a}=1, \\
c_{2}=\lim _{a \rightarrow \infty}\left(t^{I F}-c_{1} a\right)=\lim _{a \rightarrow \infty} \sqrt{a^{2}+a c}-a= \\
=\lim _{a \rightarrow \infty} \frac{a c}{\sqrt{a^{2}+a c}+a}=\frac{c}{2}=\frac{b-p-v}{2 k M}, \\
\text { that is } t=a+\frac{b-p-v}{2 k M} .
\end{gathered}
$$

Determined principle view of the dependence $t^{I F}(a)$ is shown in Fig. 3.

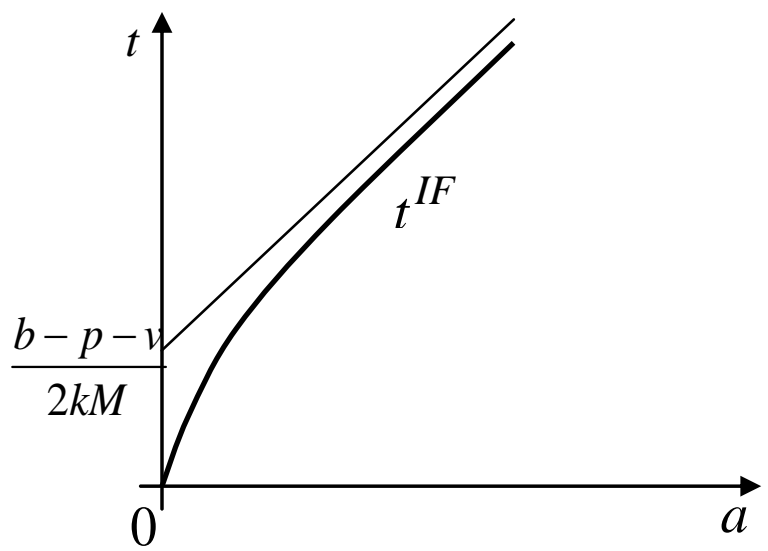

Fig. 3. Principal view of the total transportation and sale time's $t^{I F}$ dependence on conditionally constant time of its transportation and sale $a$

On the other hand, it is easy to see that $t^{I F}$ as a function of $\boldsymbol{M}$ when fixed $\boldsymbol{a}$ comes down, convex and has horizontal asymptote $\quad t=a$

(Fig.

$4)$.

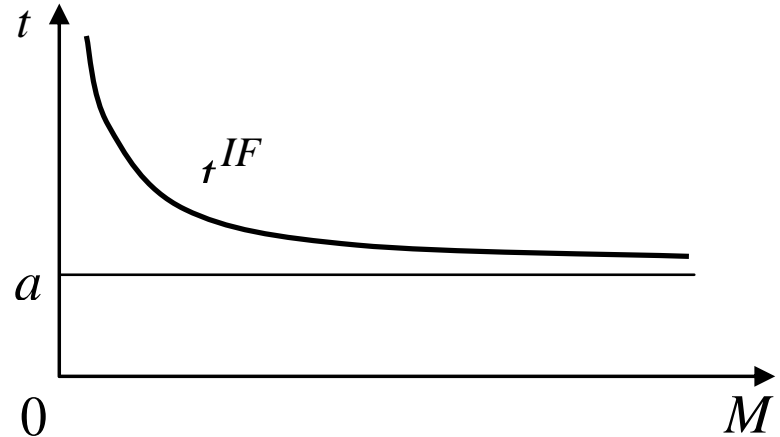

Fig. 4. Principal view of the total transportation and sale time's $t^{I F}$ dependence on transport capacity $\boldsymbol{M}$

Finally, the actual intensity of profits (producer's benchmark indicator) $I_{1}^{I F}=\frac{F^{I F}}{t^{I F}}=\frac{F_{1}^{I F}}{a+\frac{Q^{I F}}{M}}$ slower comes on $a$ for this $M$, and $\lim I_{1}^{I F}=0$ $a \rightarrow \infty$

(Fig. 5), and slower increases in $M$ with given $a$, $\lim _{M \rightarrow \infty} I_{1}^{I F}=\frac{(b-p-v)^{2}}{4 a k}$ (Fig. 6).

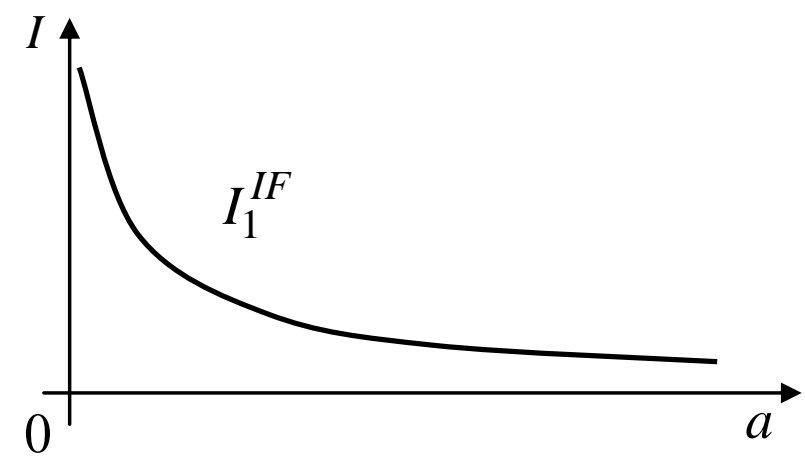

Fig. 5. Principal view of the manufacturer profit's inte $I_{1}^{I F}$ dependence on conditionally constant time of its transportation and sale $a$

Let us substitute now the function of optimal (by criterion of profit's intensity) producer's reaction (12) on the transport tariff $p$ in objective function of transport company (2), we obtain $\quad F_{2}=(p-z) \cdot Q^{I F}$

$$
=(p-z) \cdot\left(\sqrt{a^{2} M^{2}+\frac{a M(b-p-z)}{k}}-a M\right) \rightarrow \max _{p}
$$

We find equilibrium transport tariff $p^{I F}$ by equating to zero derivative $\frac{d F_{2}}{d p}$ and solving appropriate equation: 


$$
\begin{gathered}
p^{I F}=z+\frac{1}{9}(6(b-p-v)+4 k a M)- \\
-\frac{1}{9} \sqrt{(6 b-6 v+3 z+4 k a M)^{2}-36(b-v+k a M)(b-v+z)-9 z^{2}}
\end{gathered}
$$

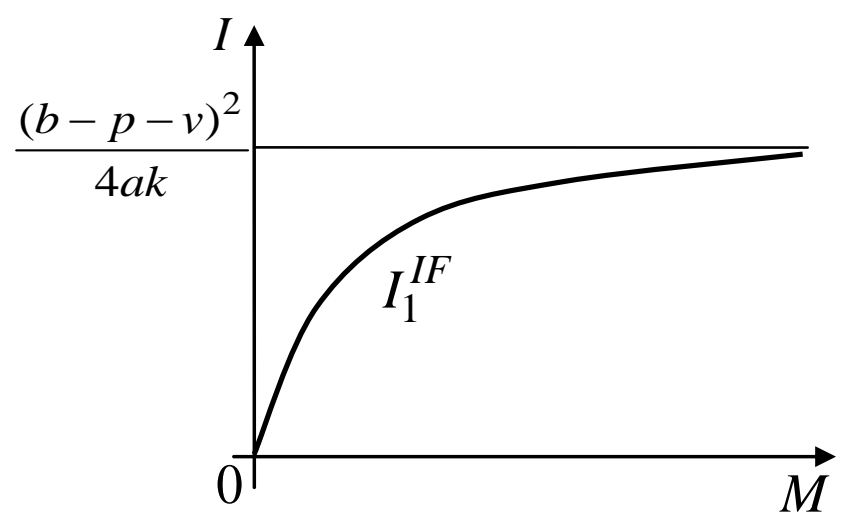

Fig. 6. Principal view of the manufacturer profit's intensity $I_{1}^{I F}$ dependence on transport capacity $M$

It is interesting that this transport tariff provides the same ratio of equilibrium participants' profits $F_{2}^{I F}=2 \cdot F_{1}^{I F}$, as was the case when they maximize their own profits.

Let us explore the asymptotic behavior of equilibrium transport

tariff.

$\lim _{a \rightarrow 0} p^{I F}=\lim _{M \rightarrow 0} p^{I F}=z+\frac{2(b-v-z)}{3}=z+\frac{2}{3} A$,

$\lim _{a \rightarrow \infty} p^{I F}=\lim _{M \rightarrow \infty} p^{I F}=z+\frac{(b-v-z)}{2}=z+\frac{1}{2} A=p^{F F}$.

Therefore, equilibrium transport tariff depending on values $a$ and $M$ includes the cost of transportation $Z$ and from half to two-thirds of the economic potential of production and transport chain $A$.

With the growth of conditionally constant expenses on organization of transportation and with increasing of transport capacity equilibrium transport tariff comes down and slower, approaching equilibrium tariff in situation where both participants of the production and transport system maximize their profits (Fig. 7).

\section{VIII.CONCLUSIONS}

Thus, taking into account the factor of time (as part of the criterion of profit's intensity) leads to increasing of equilibrium transport tariffs, reducing of the products batch volume and of the profit from it. However, with increasing of conditionally constant costs for organization of transportation and transport capacity impact of time's factor disappears, and the equilibrium conditions of production and transport system for different criteria of producer's optimality are the same.

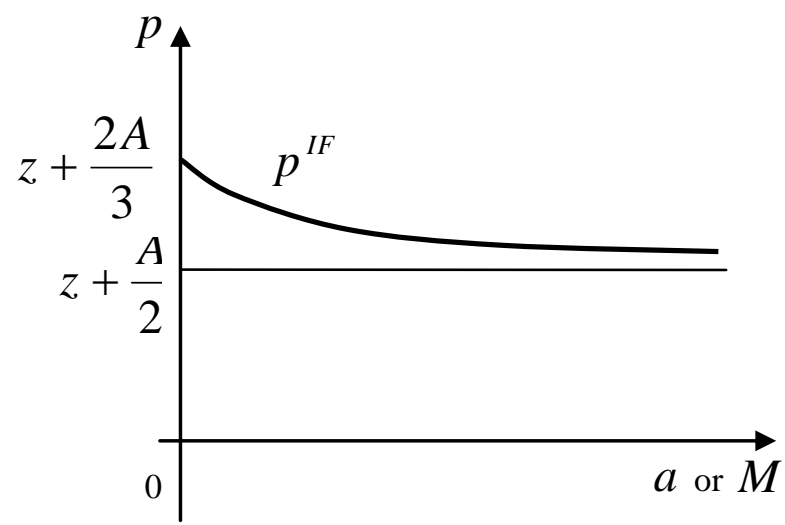

Fig. 7. Principal view of the equilibrium transport tariff's $p^{I F}$ dependence on conditionally constant time for organization of product's transportation and sale $a$ and on transport capacity $M$

\section{REFERENCES}

[1] M. G. Speranza, "Trends in transportation and logistics", Europ J.Oper. Res., no. 264(3), pp.830-836, 2018.

[2] D. Simchi-Levi, X. Chen, and J. Bramel, The Logic of Logistics: Theory, Algorithms, and Applications for Logistics and Supply Chain Management, Springer / Springer Series in Operations Research and Financial Engineering, 2004.

[3] J .Shapiro, Modeling the Supply Chain, Duxbury Press, 2000.

[4] C.Poirier, Using Models to Improve the Supply Chain, CRC Press, 2003.

[5] H. Stadtler, and C. Kilger, Supply Chain Management and Advanced Planning: Concepts, Models, Software and Case Studies, SpringerVerlag Telos, 2004.

[6] D. Simchi-Levi, D. Wu, and Z. Shen, Handbook of Quantitative Supply Chain Analysis: Modeling in the E-Business Era, Springer / International Series of Operations Research and Management Science, 2004.

[7] S. Voss, D. Woodruff, Introduction to Computational Optimization Models for Production Planning in a Supply Chain, Springer-Verlag, 2003.

[8] J. Geunes, P. Pardalos, and H. Romeijn, Supply Chain Management: Models, Applications, and Research Directions, Kluwer Academic Publishers, 2002.

[9] H. R.Varian, Microeconomic Analysis, W.W.Norton \& Company, 1992.

[10] G. Jehle, and Ph. Reny, Advanced Microeconomic Theory, Addison Wesley, 2000

[11] S. Mathis, J. Koscianski, Microeconomic Theory: An Integrated Approach, Prentice Hall, 2002.

[12] W. Nicholson, Intermediate Microeconomics and its Application, Harcourt, 2000.

[13] D. Carlton, J. Perloff, Modern Industrial Organization, Addison Wesley, 2004

[14] R. Schmalensee, and R. Willig, Handbook of Industrial Organization, Vol.1, Elsevier Science, 2002.

[15] J. Church, and R. Ware, Industrial Organization. A Strategic Approach, McGraw-Hill, 2000.

[16] L. Pepall, D. Richards, and G. Norman, Industrial Organization: Contemporary Theory and Practice, South-Western College Publishing, 2001.

[17] M. Voynarenko, and A. Kholodenko, "Profit intensity criterion for transportation problems", Global. J.Environ. Sci. Manage., no. 5(SI), pp.131-140, 2019. Doi: 10.22034/gjesm.2019.SI.15, 